\title{
Soft Tissue Attenuation Patterns Associated with Supine Acquisition SPECT Myocardial Perfusion Imaging: A Descriptive Study
}

\author{
Rami Doukky ${ }^{1, *}$, Mouyyad Rahaby ${ }^{2}$, Tareq Alyousef ${ }^{2}$, Raj Vashistha ${ }^{2}$, Dave Chawla ${ }^{3}$ and Amit P. Amin ${ }^{4}$ \\ ${ }^{I}$ Section of Cardiology, Nuclear Cardiology and Stress Testing Laboratories, Rush University Medical Center, Chicago, \\ IL, USA \\ ${ }^{2}$ Division of Adult Cardiology, John H. Stroger, Jr. Hospital of Cook County, Chicago, IL, USA \\ ${ }^{3}$ Section of Cardiology, Rush University Medical Center, Chicago, IL, USA \\ ${ }^{4}$ Division of Cardiology, John H. Stroger, Jr. Hospital of Cook County, Chicago, IL, USA
}

\begin{abstract}
Purpose: Soft-tissue attenuation patterns in SPECT-myocardial perfusion imaging (MPI) of supine acquisition systems are well recognized. Their prevalence and interaction with body-habitus and gender are ill-defined, which we sought to describe in this study.

Methods: In a cross-sectional study, we described the prevalence of soft-tissue attenuation patterns in normal SPECT-MPI studies acquired with a supine patient-position SPECT system.

Results: In 263 normal, clinically-indicated, supine-acquisition SPECT-MPIs the attenuation patterns observed were: anterior $(35.4 \%)$, inferior $(41.8 \%)$ and lateral $(13.3 \%)$. Anterior attenuation was more prevalent among women $(50.7 \%$ vs. $15.7 \%, \mathrm{P}<0.001)$ and was associated with chest circumference among men. Conversely, inferior attenuation was more prevalent among men $(78.3 \%$ vs. $13.5 \%, \mathrm{P}<0.001)$ and was not affected by body-habitus. Lateral attenuation was more common among women $(19.6 \%$ vs. $5.2 \%, \mathrm{p}=0.001)$ and was associated with obesity $(\mathrm{p}=0.015)$.

Conclusions: Soft-tissue attenuation artifacts are common in supine-acquisition SPECT-MPI. The recognition of their prevalence and association with body-habitus and gender is critical for the accurate interpretation of SPECT-MPI.
\end{abstract}

Keywords: SPECT, myocardial perfusion imaging, soft tissue attenuation, artifacts, supine, SPECT acquisition, interpretation.

\section{INTRODUCTION}

Soft tissue attenuation remains problematic for myocardial perfusion imaging (MPI) with Single Photon Emission Computed Tomography (SPECT) [1]. Hybrid SPECT-Computed Tomography (CT) systems address attenuation correction very effectively [2-4], but they remain expensive and are not commonly used outside large centers [5], Therefore, practicing physicians are likely to continue to interpret non-corrected SPECT-MPI for years to come . Therefore, the recognition of attenuation patterns associated with SPECT-MPI is important for accurate MPI interpretation [6]. Fortunately, practicing physicians are well versed with the attenuation patterns associated with supine acquisition SPECT-MPI [6]. However, their prevalence and interaction with gender and body habitus are not well described in the literature. In a previous report, we compared the attenuation patterns observed in supine vs. upright acquisition [7]. In this investigation, we further examined the association of the attenuation patterns seen in supine imaging with gender and body habitus.

*Address correspondence to this author at the Rush University Medical Center, Section of Cardiology, 1653 W. Congress Pkwy, Chicago, IL 60612, USA; Tel: 312-563-2534; Fax: 312-942-6334;

E-mail: rami_doukky@rush.edu

\section{METHODS}

We conducted a retrospective cross-sectional evaluation of 602 consecutive clinically-indicated outpatient SPECTMPI studies performed between August 2007 and July 2008 using a supine-acquisition cardiac-SPECT system (Siemens dual-head, 90-degree ECAM), which "passed" a SPECT phantom study conformed to ACR guidelines [8], scoring "satisfactory" for spatial resolution, uniformity and contrast. A one-day Technetium- $99 \mathrm{~m}$ tetrofosmin protocol was implemented [9]. Stress and acquisition protocols conformed to ASNC guidelines [10-12]. Women were imaged without a bra. A Butterworth post-processing filter of order 5, cut-off $0.6 \mathrm{cycle} / \mathrm{cm}$ was applied to the three dimensional reconstructed volume. Image processing and analysis was performed with 4DM-SPECT software (INVIA - Ann Arbor, MI).

To isolate attenuation artifacts from perfusion abnormalities we evaluated only normal MPI studies in a population with a low prevalence of coronary artery disease (CAD). First, we excluded subjects with clinically established CAD or typical angina [13]. Second, all MPI scans were compared quantitatively to a database of normal MPI scans from individuals with a low likelihood of CAD obtained on an identical camera. All subjects with a summed stress score $(\mathrm{SSS})>2$, summed difference score $(\mathrm{SDS})>0$ and those with quantitatively identified wall motion 
abnormalities were excluded. Consequently, we were left with 263 subjects. Third, we confirmed that the remaining group of patients were at low CAD risk; as the calculated 10year Framingham coronary heart disease risk [13] was relatively low $[13.2 \%( \pm 10.4)]$ and the mean probability of obstructive CAD among symptomatic patients [14] was also low $[14.36 \%( \pm 10.3)]$. By this 3-step process, we ensured that our study population had a low likelihood of CAD and quantitatively normal MPI scans. We thus had a high level of confidence that the vast majority of MPI scans included in the analysis were "truly" normal. Therefore, we considered any reduced activity identified by subsequent analysis to be due to attenuation rather than true perfusion abnormality.

In the absence of a standard definition of an attenuation defect, we adopted a methodology to define them in a dichotomous fashion. Attenuation defects were classified a priori, based on their locations on a 17-segment model [12] to anterior, inferior and lateral, as defined in Fig. (1). Only stress-SPECT images were analyzed quantitatively to identify attenuation defects. The quantitative analysis was performed using a 17-segment polar map display of photon count statistics normalized in percentage to the highest count pixel. An attenuation defect was considered to be "present" if the normalized count statistics in one or more contiguous myocardial segments was decreased by more than 20 percentage points from that of the highest activity segment in the plot. Normalized count percentages in all contiguous segments constituting one defect were averaged and tabulated. The defect was assigned a classification (anterior, inferior or lateral) based on the location of the segment of minimum activity. The detailed methodology used in this study was published elsewhere [7]. The "density" of an attenuation defect was considered to be the inverse of normalized count statistics of photon activity; as the lower the normalized count statistics in a defect was, the denser the attenuation.

The chi-square test was used to compare the prevalence of attenuation defects between various subgroups. Multivariable logistic regression models were used to analyze the independent factors associated with various attenuation defects. The rates of observed defects were reported as proportions and odds-ratios (OR) with 95\% confidence intervals $(95 \% \mathrm{CI})$. Linear correlation between normalized count statistics in a defect, BMI and chest circumference was investigated using Pearson's method. Only two-tailed p-values were reported. The study was internally funded and approved by the institutional review board of Rush University Medical Center.

\section{RESULTS}

We analyzed 263 subjects. The baseline characteristics of the study population are summarized in Table 1 . The prevalence of attenuation patterns observed in the entire study population and in each gender group is detailed in Table 2.

Anterior attenuation was observed in 93 (35.4\%) patients and was significantly more common among women; seen in 75 women $(50.7 \%)$ vs. 18 men $(15.7 \%) \quad[\mathrm{OR}=5.5$, 95\% CI $=3.0-10.1, \mathrm{p}<0.001]$ (Table 2, Fig. 2). Additionally, there was no association between anterior attenuation and BMI $(p=0.13)$. Further, there was a trend toward an association between anterior attenuation and chest circumference $(p=0.07)$ with an apparent differential effect of gender by univariate analysis; as men with anterior attenuation trended toward having a larger chest circumference $(114.6 \mathrm{~cm})$ than those without anterior attenuation $(110.3 \mathrm{~cm}), \mathrm{p}=0.09$. Whereas, among women there was no significant difference in chest circumference between those with or without anterior attenuation $(\mathrm{p}=$ 0.22). Multivariable logistic regression analysis identified female gender to be the only factor associated with anterior attenuation, controlling for BMI and chest size (OR: 10.05, 95\% CI=3.8-26.8, $\mathrm{p}<0.001)$, as summarized in Table $\mathbf{3}$. Additionally, there was no interaction between gender and chest circumference or BMI on anterior attenuation (interaction- $p=0.7$ and 0.6 , respectively). When we studied men alone in a multivariable regression model chest circumference was predictive of anterior attenuation independent of $\mathrm{BMI}(\mathrm{OR}=1.09$ for each $1 \mathrm{~cm}$ increase in chest circumference, $95 \% \mathrm{CI}=1.002-1.2, \quad \mathrm{p}=0.04)$; confirming the trend observed in univariate analysis. Finally, we inspected SPECT and rotating planar images looking for shifting anterior attenuation resulting from a change in breast position between the resting and stress acquisitions. There

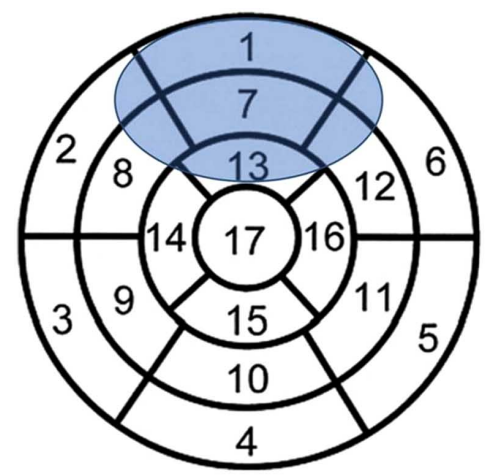

A. Anterior Wall Attenuation

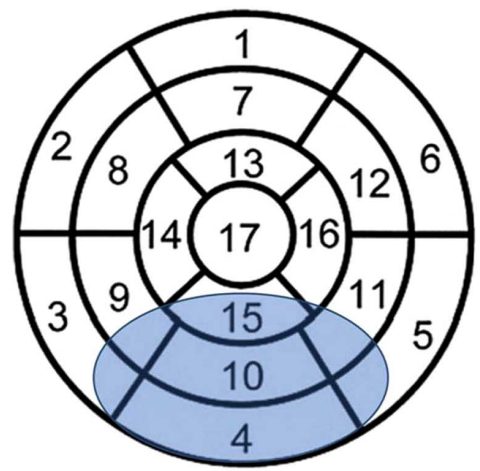

B. Inferior Wall Attenuation

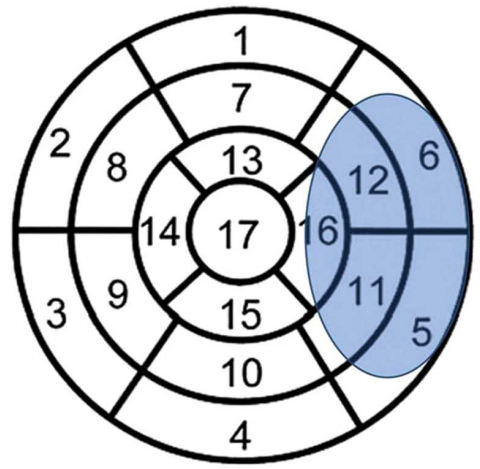

C. Lateral Wall Attenuation

Fig. (1). Definition of attenuation defects. A. Anterior wall attenuation was defined as reduced activity in the anterior segments with or without involvement of the anterolateral and/or anteroseptal segments. B. Inferior wall attenuation was defined as reduced activity in the inferior segments with or without involvement of the inferoseptal and/or inferolateral segments. C. Lateral wall attenuation was defined as reduced activity in the inferolateral and/or anterolateral segments, but sparing the anterior and inferior segments. Reprinted with permission (Chawla D et al. J Nucl Cardiol. 2011 [7]). 
Table 1. Baseline Characteristics

\begin{tabular}{|c|c|}
\hline Number of subjects & 263 \\
\hline Female & $148(56.3 \%)$ \\
\hline Age (mean) & $56.62( \pm 14.1)$ \\
\hline Hypertension & $63.3 \%$ \\
\hline Diabetes Mellitus & $22.0 \%$ \\
\hline Dyslipidemia & $50.0 \%$ \\
\hline Smoking & $13.7 \%$ \\
\hline Family History of CAD & $35.2 \%$ \\
\hline Mean chest circumference $(\mathbf{c m})$ & $103.1( \pm 11.7)$ \\
\hline Male & $111.0( \pm 9.7)$ \\
\hline Female & $96.9( \pm 9.3)$ \\
\hline Mean BMI $\left(\mathrm{Kg} / \mathrm{m}^{2}\right)$ & $31( \pm 7.2)$ \\
\hline Male & $30.7( \pm 5.1)$ \\
\hline Female & $31.7( \pm 8.2)$ \\
\hline Exercise Stress & $156(59.3 \%)$ \\
\hline CAD likelihood * & $14.36( \pm 10.3)$ \\
\hline Framingham 10-year CHD risk & $13.2( \pm 10.4)$ \\
\hline Mean Summed Stress Score (SSS) & 0.60 \\
\hline Ejection Fraction (Gated SPECT) & $66.3 \%( \pm 4.0)$ \\
\hline
\end{tabular}

CAD: Coronary artery disease; BMI: Body Mass Index; CHD: coronary Heart Disease. *Relevant to symptomatic patients only and was based on Diamond and Forrester prediction tables [13]

were $9(3.4 \%, 95 \% \mathrm{CI}=1.2-5.6)$ cases of shifting anterior attenuation ( 2 definite and 7 probable).

There was a very modest linear correlation between the density of anterior attenuation and BMI [Pearson's correlation coefficient $(r)=0.21, p=0.04$ ] (Table 3). In other words; the higher the BMI was, the "denser" the anterior attenuation. There was no correlation between anterior attenuation and chest circumference $(\mathrm{p}=0.91)$.

Inferior attenuation was very common and was observed in $110(41.8 \%)$ subjects and was more prevalent among men [90 (78.3\%)] vs. women [20 (13.5\%)], OR=23.0, 95\%CI=
$12.1-44.0, \mathrm{p}<0.001$. (Table 2, Fig. 2). There was no association between inferior attenuation and BMI $(p=0.44)$. Univariate analysis showed that inferior attenuation was associated with larger chest circumference $(109.9 \mathrm{~cm}$ vs. $99.1 \mathrm{~cm}), \mathrm{p}<0.001$. However, multivariable logistic regression analysis showed that male gender was the only independent factor associated with inferior attenuation, $(\mathrm{OR}=23.3$, 95\% CI $=7.9-66.7, \quad \mathrm{p}<0.001)$; whereas BMI and chest circumference were not associated with inferior attenuation when studied in the same model $(p=0.74$ and 0.93 , respectively). This suggests that chest circumference is merely a confounder as men had larger chests, and only male gender is the true independent factor associated with inferior attenuation. There was no correlation between the density of inferior attenuation and BMI or chest circumference $(p=0.57$ and 0.29 , respectively), as shown in Table $\mathbf{3}$.

Lateral attenuation was least common and was observed in 35 subjects $(13.3 \%)$. It had a strong univariate association with female gender: observed in $29(19.6 \%)$ women vs. 6 $(5.2 \%)$ men $(\mathrm{OR}=4.4,95 \% \mathrm{CI}=1.8-11.1), \mathrm{p}=0.001$ (Table 2, Fig. 2). Additionally, it was associated with obesity (BMI $\geq 30)$ : observed in $24(18.5 \%)$ obese vs. $11(8.3 \%)$ non-obese subjects $(\mathrm{OR}=2.5,95 \% \mathrm{CI}=1.2-5.4), \mathrm{p}=0.015$; with a trend toward a differential effect of female gender (MantelHaenszel's p-value $=0.06$ ). The association between marked obesity and lateral attenuation was even stronger: observed in $20(23.8 \%)$ subjects with $\mathrm{BMI} \geq 33$ vs. $15(8.4 \%)$ among those with $\mathrm{BMI}<33(\mathrm{OR}=3.4,95 \% \mathrm{CI}=1.6-7.1, \mathrm{p}=0.001)$ with a convincing differential effect of female gender (Mantel-Haenszel's p-value $=0.004$ ). On the other hand, there was no significant difference in chest circumference among those with vs. without lateral attenuation $(p=0.39)$ without a differential effect of gender (Table 3). When tested in a multivariable logistic regression model, female gender was an independent predictor of lateral attenuation $(\mathrm{OR}=4.2$, $95 \% \mathrm{CI}=1.3-13.8, \mathrm{p}$-value $=0.02)$, adjusting for obesity (BMI $\geq 30)$ and chest size. Additionally, marked obesity (BMI $\geq 33$ ) was associated with lateral attenuation independent of gender and chest circumference $(\mathrm{OR}=3.2,95 \% \mathrm{CI}=1.3-$ 8.6, $\mathrm{p}=0.01$ ) (Table 3). Furthermore, no interaction was found between obesity and female gender on lateral attenuation (interaction p-value $=0.79$ ). Among men, lateral attenuation was rare ( 6 subjects, $5.2 \%$ ) and, therefore, the study was underpowered to detect any significant association with body habitus.

Finally, There was no correlation between the density of lateral attenuation and BMI or chest circumference $(p=0.84$ and 0.66 , respectively), as shown in Table 3 .

Table 2. Rates of Attenuation Patterns Observed

\begin{tabular}{|l|c|c|c|c|}
\hline Attenuation & $\begin{array}{c}\text { Total, } \boldsymbol{n = 2 6 3} \\
\text { Percent } \pm \mathbf{9 5 \%} \text { CI }\end{array}$ & $\begin{array}{c}\text { Men, } \boldsymbol{n = 1 1 5} \\
\text { Percent } \pm \mathbf{9 5 \%} \text { CI }\end{array}$ & $\begin{array}{c}\text { Women, } \boldsymbol{n}=\mathbf{1 4 8} \\
\text { Percent } \pm \mathbf{9 5 \%} \text { CI }\end{array}$ & $\begin{array}{c}\text { Chi-square } \boldsymbol{p} \text {-value } \\
\text { Men vs. Women }\end{array}$ \\
\hline \hline Anterior & $93(35.4 \pm 5.8 \%)$ & $18(15.7 \pm 6.6 \%)$ & $75(50.7 \pm 8.1 \%)$ & $<0.001$ \\
\hline Inferior & $110(41.8 \pm 6.0 \%)$ & $90(78.3 \pm 7.5 \%)$ & $20(13.5 \pm 5.5 \%)$ & $<0.001$ \\
\hline Lateral & $35(13.3 \pm 4.1 \%)$ & $6(5.2 \pm 4.1 \%)$ & $29(19.6 \pm 6.4 \%)$ & 0.001 \\
\hline
\end{tabular}

95\% CI: $95 \%$ confidence interval. 


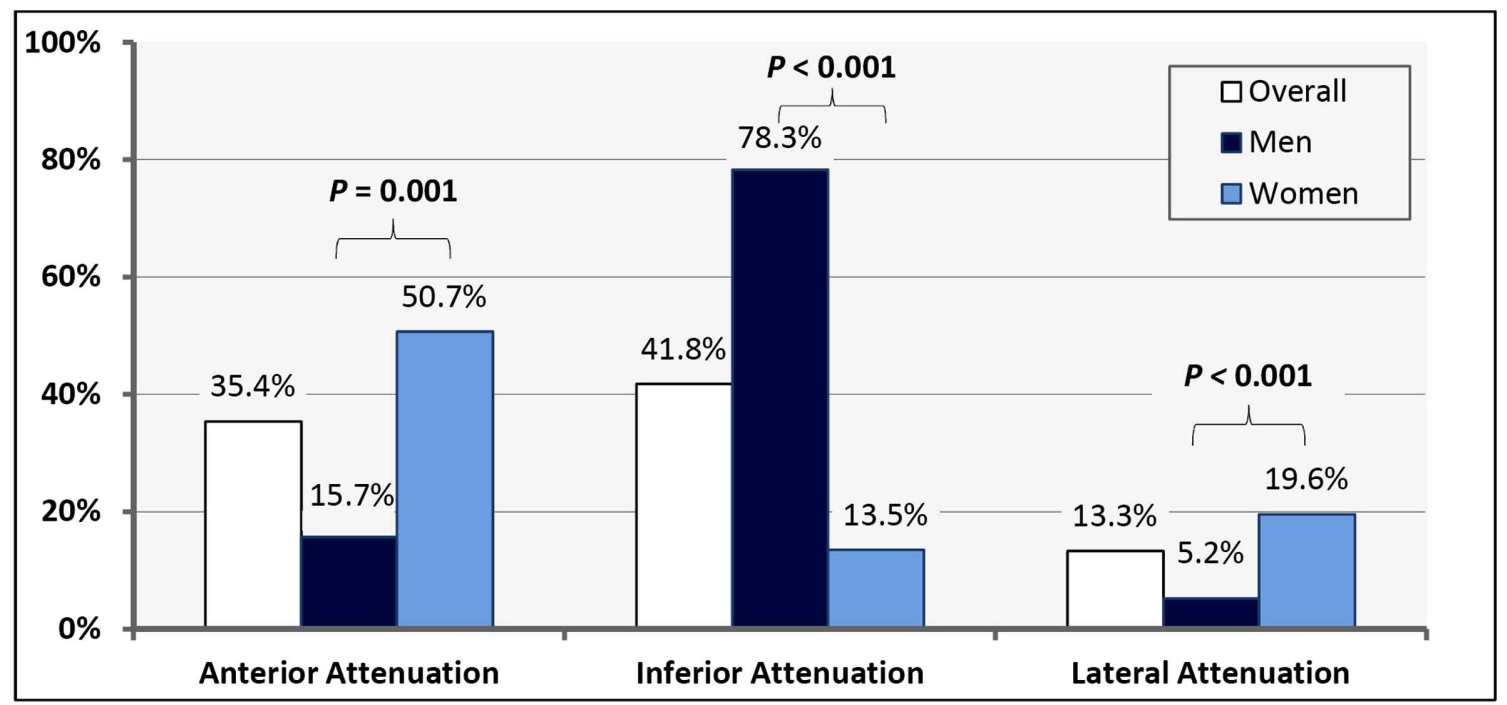

Fig. (2). Attenuation Patterns Observed.

Table 3. Characteristics of the Attenuation Patterns Observed

\begin{tabular}{|l|c|c|c|c|c|}
\hline Attenuation Type & Prevalence & Gender & $\begin{array}{c}\text { Effect of Chest Size on } \\
\text { Prevalence }\end{array}$ & $\begin{array}{c}\text { Effect of BMI on } \\
\text { Prevalence }\end{array}$ & $\begin{array}{c}\text { Factors that Increase } \\
\text { Severity }\end{array}$ \\
\hline \hline Anterior & Common & F > M & - in M \\
- in F & BMI \\
\hline Inferior & Most common & M > F & - & - & None \\
\hline Lateral & Least common & F > M & - & None \\
\hline
\end{tabular}

\section{DISCUSSION}

The value of SPECT-MPI for the diagnosis and risk stratification of CAD is well-established (14). However, the full clinical potential of SPECT-MPI is often compromised due to numerous artifacts that degrade image quality and increase the chance of image misinterpretation (15). Soft tissue attenuation is the most frequent source of artifacts in SPECT-MPI since the heart is surrounded by tissues of varying densities and attenuation coefficients (e.g., bone, diaphragm, lungs, and breast). Thus non-uniform attenuation of myocardial photon activity is often encountered in SPECT-MPI, resulting in various patterns of attenuation artifacts depending on many factors such as: body size, depth of the heart in the body, gender, and patient position. Recognizing these attenuation patterns is crucial to optimizing the diagnostic accuracy of SPECT-MPI; as misinterpretation may lead to unwarranted downstream testing, including cardiac catheterization, at an added risk and expense.

The supine patient position SPECT-MPI acquisition are the most widely used systems to acquire cardiac MPI images, so we designed this study to comprehensively describe the attenuation patterns observed with supine SPECT-MPI and their interaction with body habitus and gender. We summarize our findings in three salient points: First, anterior attenuation was very common, particularly among women. BMI was not predictive of anterior attenuation in men or women. In the entire cohort, chest circumference trended toward being larger among those with anterior attenuation, with an apparent differential effect of gender; as among men chest circumference was positively associated with anterior attenuation, independent of BMI. Counter-intuitively, chest circumference was not associated with anterior attenuation among women. The density of the anterior attenuation had only modest correlation with BMI and no correlation with chest circumference. Furthermore, this study confirms that change in female breast position is encountered in patients undergoing supine acquisition SPECTMPI, which can occasionally produce the "appearance" of a reversible anterior perfusion defect. Second, inferior attenuation was also common particularly among men and was independent of BMI and chest circumference. Third, lateral attenuation was least commonly observed and was independently associated with female gender and obesity, but not chest circumference. We explain this finding by possible attenuation from thick folds of skin and soft tissue covering the lateral chest wall in short, obese women. Among men, lateral attenuation was too rare to draw any conclusions regarding its interaction with body habitus. Lastly, there was no correlation between the density of inferior or lateral attenuation and BMI or chest circumference.

Our study has certain limitations. First, we acknowledge the limitation of the retrospective nature of our investigation. However, there is little to be gained from a prospective design; besides that it is highly unlikely to be ever conducted. Second, we did not have an independent reference gold-standard to confirm that all scans evaluated were truly normal. However, we demonstrated that all scans 
were quantitatively normal in a population with low CAD risk. Third, we included scans with SSS of 1 or 2 (but SDS of 0 ) as they are likely to be normal given a normal segmental wall motion and low CAD prevalence; and by excluding subjects with SSS of 1 or 2 we are likely to eliminate patients with prominent attenuation artifacts but normal perfusion. Finally, this study may be underpowered to detect certain interactions.

\section{CONCLUSIONS}

Soft-tissue attenuation artifacts were common in supine-acquisition SPECT-MPI. Anterior attenuation was particularly common among women; observed in 1 out of 2 females. Anterior attenuation was uncommon among men but seems to be associated with larger chest circumference. Inferior attenuation was very frequent among men; affecting 4 out 5 men. Lateral attenuation was least common and was strongly associated with obesity and female gender; affecting 1 out of 5 women and 1 out of 4 markedly obese women. Recognizing the frequency of these attenuation patterns and their relation to gender and body habitus is critical for the accurate interpretation of SPECT-MPIs acquired with supine imaging systems.

\section{CONFLICT OF INTEREST}

Declared none.

\section{ACKNOWLEDGEMENT}

Declared none.

\section{REFERENCES}

[1] Hendel RC. Attenuation correction: eternal dilemma or real improvement? Q J Nucl Med Mol Imaging 2005; 49: 30-42.
[2] Bybel B, Brunken RC, DiFilippo FP, Neumann DR, Wu G, Cerqueira MD. SPECT/CT imaging: clinical utility of an emerging technology. Radiographics 2008; 28: 1097-113.

[3] Garcia EV. SPECT attenuation correction: an essential tool to realize nuclear cardiology's manifest destiny. J Nucl Cardiol 2007; 14: 16-24.

[4] Singh B, Bateman TM, Case JA, Heller G. Attenuation artifact, attenuation correction, and the future of myocardial perfusion SPECT. J Nucl Cardiol 2007; 14: 153-64.

[5] IMV Medicine Market Summary Report, Des Plaines IL: IMV Medical Information Division 2008.

[6] Hendel RC. Interpretation of Myocardial Perfusion Imaging. In: Heller GV, Hendel RC, Eds. Nuclear Cardiology: Practical Applications. New York: McGraw-Hill 2004: 157-74.

[7] Chawla D, Rahaby M, Amin AP, et al. Soft tissue attenuation patterns in stress myocardial perfusion SPECT images: a comparison between supine and upright acquisition systems. J Nucl Cardiol 2011; 18: 281-90.

[8] American College of Radiology Nuclear Medicine Accreditation Program. Site Scanning Instructions for ACR Nulcear Medicine Phantom 2010; Available http: //www.acr.org/accreditation/ nuclear/qc_forms/FeaturedCategories/qc_forms/SPECT_Phantom_ Instructions.aspx.

[9] Henzlova MJ, Cerqueira MD, Mahmarian JJ, Yao SS. Stress protocols and tracers. J Nucl Cardiol 2006; 13: e80-90.

[10] Abbott BG. Stress Testing In: Mann A, Heller GV, Hendel RC, Eds. Nuclear Cardiology: Technical Applications. New York: McGraw-Hill 2007: 97-106.

[11] Doukky R. Pharmacologic Stress testing in myocardial perfusion imaging: Technical applications. In: Mann A, Heller GV, Hendel RC, Eds. Nuclear Cardiology: Technical Applications. New York: McGraw-Hill 2007; 107-24.

[12] Hansen CL, Goldstein RA, Berman DS, et al. Myocardial perfusion and function single photon emission computed tomography. J Nucl Cardiol 2006; 13: e97-120.

[13] Diamond GA, Forrester JS. Analysis of probability as an aid in the clinical diagnosis of coronary-artery disease. N Engl J Med 1979; 300: 1350-8.

[14] Wilson PW, D'Agostino RB, Levy D, Belanger AM, Silbershatz H, Kannel WB. Prediction of coronary heart disease using risk factor categories. Circulation 1998; 97: 1837-47. 